\title{
Ação de produtos fitossanitários utilizados em cafeeiros sobre pupas e adultos de Chrysoperla externa (Hagen, 1861) (Neuroptera: Chrysopidae)
}

\author{
Action of pesticides used in coffee crops on the pupae and adults of Chrysoperla externa (Hagen, 1861) \\ (Neuroptera: Chrysopidae)
}

\author{
Rogério Antônio Silva ${ }^{1}$ Geraldo Andrade Carvalho ${ }^{2}$ César Freire Carvalho ${ }^{2}$ Paulo Rebelles Reis ${ }^{1}$ \\ Brígida Souza ${ }^{2}$ Antônio Marcos Andrade Rezende Pereira ${ }^{3}$
}

\section{RESUMO}

Avaliou-se a ação de produtos fitossanitários usados em cafeeiros sobre pupas e adultos de Chrysoperla externa (Hagen, 1861) (Neuroptera: Chrysopidae). Os bioensaios foram conduzidos no Departamento de Entomologia da Universidade Federal de Lavras - UFLA, Lavras, MG, Brasil. Os tratamentos avaliados, em g i.a. $L^{-1}$ de água, foram: 1endosulfan (Thiodan 350 CE - 1,75), 2- chlorpyrifos (Lorsban $480 C E$-1,2), 3- betacyfluthrin (Turbo $50 C E-0,013), 4-$ enxofre (Kumulus 800 PM - 4,0), 5- azocyclotin (Peropal 250 PM - 0,31), 6- oxicloreto de cobre (Cuprogarb 500 PM - 5,0) e 7- Testemunha (água). As pulverizações foram realizadas diretamente sobre pupas e adultos do crisopídeo por meio de torre de Potter. As pupas foram colocadas em tubos de vidro e os adultos em gaiolas de PVC, e mantidos em sala climatizada a $25 \pm 2{ }^{\circ} \mathrm{C}, \mathrm{UR}$ de $70 \pm 10 \%$ e fotofase de 12 horas. $O$ delineamento experimental foi inteiramente casualizado, com sete tratamentos e dez repetições, sendo cada parcela formada por quatro pupas ou um casal de C. externa. Os produtos foram distribuídos nas quatro classes de toxicidade conforme escala estabelecida pela IOBC. O chlorpyrifos mostrou-se levemente nocivo para pupas (classe 2, $30 \leq E \leq 79 \%$ ), e os demais produtos foram inócuos (classe 1, E<30\%). O endosulfan, enxofre, azocyclotin e oxicloreto de cobre foram inócuos em adultos, enquanto o betacyfluthrin foi moderadamente nocivo (classe 3, 80 $\leq$ E $99 \%$ ) e o chlorpyrifos foi nocivo (classe 4, E>99\%). Os produtos testados à base de endosulfan, enxofre, azocyclotin e oxicloreto de cobre podem ser recomendados em programas de manejo de pragas do cafeeiro em associação com C. externa, em função da baixa toxidade apresentada por esses compostos ao predador.

Palavras-chave: Coffea arabica, crisopídeo, controle integrado de pragas, seletividade.

\begin{abstract}
It was evaluated the action of the pesticides endosulfan, chlorpyrifos, betacyfluthrin, sulphur, azocyclotin and copper oxychloride to pupae and adults of Chrysoperla externa (Hagen)(Neuroptera: Chrysopidae). The bioassays were carried out in the Entomology Department of the Universidade Federal de Lavras - UFLA, MG, Brazil. The treatments in $g$ i.a. $L^{-1}$ of water, were: 1 - endosulfan (Thiodan $\left.350 C E-1.75\right)$, 2 - chlorpyrifos (Lorsban $480 C E$ - 1.2), 3 - betacyfluthrin (Turbo 50 CE - 0.013), 4 - sulphur (Kumulus 800 PM - 4.0), 5 - azocyclotin (Peropal 250 PM - 0.31), 6 - copper oxychloride (Cuprogarb $500 P M-5.0)$ and 7 - control (water). The sprayings were accomplished directly on pupae and adults of green lacewing, using a Potter's tower. The pupae were placed in glass tubes and the adults in PVC cages and maintained in climatic chambers at $25 \pm 2^{\circ} \mathrm{C}, \mathrm{RH}$ of $70 \pm 10 \%$ and 12-hour photophase. The experimental design was completely randomized, with seven treatments and ten replicates. Each plot was made up by either four pupae or one couple of $\boldsymbol{C}$. externa. The pesticides were classified according recommendations of the IOBC. Chlorpyrifos was classified in the class 2 = slightly harmful $(30 \leq E \leq 79 \%)$ for pupae, and the all the other products were selective and classified in the class $1=$ harmless $(E<30 \%)$. To adults, chlorpyrifos was toxic, being classified in class $4=$ harmful $(E>99 \%)$ and betacyfluthrin in class 3 = moderately harmful $(80 \leq E \leq 99 \%)$. Endosulfan, sulphur, azocyclotin and copper oxychloride were harmless to adults, being classified in class 1. The pesticides endosulfan, azocyclotin, sulphur and copper oxychloride can be recommended in coffee pest management programs in association with $\boldsymbol{C}$. externa, based in the reduced toxicity presented in these products for this predator.
\end{abstract}

Key words: Coffea arabica, green lacewing, insect pest management, selectivity.

${ }^{1}$ Empresa de Pesquisa Agropecuária de Minas Gerais (EPAMIG-CTSM/EcoCentro), CP 176, CEP 37200-000, Lavras, MG, Brasil.

E-mail: rogeriosilva@epamig.ufla.br. Autor para correspondência.

${ }^{2}$ Departamento de Entomologia, Universidade Federal de Lavras, Lavras, MG, Brasil.

${ }^{3}$ Curso de Graduação em Agronomia da Universidade Federal de Lavras, Lavras, MG, Brasil. 


\section{INTRODUÇÃO}

No agroecossistema cafeeiro ocorrem muitas espécies de insetos que se tornam pragas de importância econômica, como o bicho-mineiro, Leucoptera coffeella (Guérin-Mèneville, 1842) (Lepidoptera: Lyonetiidae) que, muitas vezes, em condições climáticas favoráveis, ocasionam prejuízos à produção de café (REIS et al., 2002).

Dentro da filosofia do Manejo Integrado de Pragas (MIP), a conservação e o aumento de inimigos naturais que beneficiem o controle biológico natural são estratégias fundamentais (GLIESSMAN, 2000; REIS et al., 2002). Portanto, para o estabelecimento de um programa de MIP para o cafeeiro, a utilização de produtos químicos seletivos que preservem os inimigos naturais no agroecossistema faz-se necessária.

Os insetos pertencentes à família Chrysopidae têm tido importante atuação no equilíbrio da densidade populacional de muitos artrópodes-praga e dentre os crisopídeos a espécie Chrysoperla externa (Hagen, 1861) (Neuroptera: Chrysopidae) destaca-se na Região Neotropical, ocorrendo naturalmente em culturas de interesse econômico, como a do cafeeiro (Coffea spp.), sendo considerada agente potencial de controle biológico de diversas pragas de importância agrícola (CARVALHO \& SOUZA, 2000; FONSECA et al., 2001).

A fase de pupa dos crisopídeos é considerada uma das mais tolerantes a muitos produtos fitossanitários (KOWALSKA \& SZCZEPANSKA, 1988; ULHÔA et al., 2002; GODOY et al., 2004). Em relação aos adultos, algumas espécies de crisopídeos são tolerantes a determinados piretróides e altamente suscetíveis a outros e também a organofosforados (GRAFTON-CARDWELL \& HOY, 1985; GODOY et al., 2004). Levando em consideração tais fatos, o presente trabalho objetivou avaliar a ação de alguns produtos fitossanitários utilizados na cultura do cafeeiro sobre pupas e adultos de $\boldsymbol{C}$. externa.

\section{MATERIAL E MÉTODOS}

Os ensaios foram conduzidos no Laboratório de Estudos de Seletividade do Departamento de Entomologia da Universidade Federal de Lavras - UFLA de junho de 2002 a janeiro de 2003. Os compostos e doses utilizadas encontram-se relatados na tabela 1 , sendo que o tratamento testemunha foi composto somente de água.

Efeito da aplicação dos produtos em pupas.

Quarenta pupas de C. externa, por tratamento, com idade máxima de 24 horas, foram retiradas cuidadosamente de tubos de vidro de uma criação de manutenção de quarta geração do laboratório, obtidas a partir de adultos coletados em lavouras de café, no campus da UFLA. As larvas, que originaram as pupas, foram alimentadas com ovos de Anagasta kuehniella (Zeller) (Lepidoptera: Pyralidae), colocadas em placas de Petri de $15 \mathrm{~cm}$ de diâmetro por $2 \mathrm{~cm}$ de altura e pulverizadas, por meio de torre de Potter regulada à pressão de $15 \mathrm{lb} \mathrm{pol}^{-2}$, com $1,5 \pm 0,5 \mathrm{mg}$ de calda química $/ \mathrm{cm}^{2}$. Em seguida, foram individualizadas em tubos de vidro de $2,5 \mathrm{~cm}$ de diâmetro e $8,5 \mathrm{~cm}$ de altura. Esses tubos foram vedados com filme de PVC laminado e mantidos em câmara climatizada regulada com temperatura de $25 \pm 2^{\circ} \mathrm{C}$, umidade relativa de $70 \pm$ $10 \%$ e fotoperíodo com fotofase de 12 horas, para avaliações e emergência dos adultos.

O delineamento experimental foi inteiramente casualizado com sete tratamentos e dez repetições, sendo cada parcela constituída de quatro pupas. Avaliaram-se a duração e sobrevivência, através de observações diárias e a razão sexual, após a emergência dos adultos.

Avaliação da fecundidade de adultos de C. externa oriundos de pupas que receberam os tratamentos.

Os adultos emergidos de pupas tratadas foram separados por casal e individualizados em gaiolas de PVC de $10 \mathrm{~cm}$ de altura e $10 \mathrm{~cm}$ de diâmetro, revestida internamente com papel filtro. As gaiolas foram apoiadas em bandejas plásticas de $15 \mathrm{~cm}$ de diâmetro forradas com o mesmo tipo de papel, tendo a parte superior fechada com tecido tipo voil. Os adultos de C. externa foram alimentados com dieta à base de lêvedo de cerveja e mel $(1: 1 \mathrm{v} / \mathrm{v})$, conforme metodologia de BARBOSA et al. (2002).

O delineamento experimental foi $\mathrm{o}$ inteiramente casualizado com sete tratamentos e sete repetições, sendo cada parcela composta por um casal. Durante quatro semanas consecutivas, realizaram-se a contagem do número de ovos postos e separados 96 ovos por tratamento. Esses ovos foram individualizados em compartimentos de placas de microtitulação usadas em teste ELISA(Enzime Linked Immunosorbent Assay), fechadas com PVC laminado e mantidas em sala climatizada nas mesmas condições descritas anteriormente. Avaliou-se a capacidade diária (média de cada três dias) e total de oviposição por fêmea, nas quatro semanas consecutivas, bem como a viabilidade dos ovos.

Análise dos dados.

O efeito total (E em \%, efeito na mortalidade e reprodução) de cada produto testado ao longo da fase adulta do predador foi determinado por meio da 
Tabela 1 - Especificação dos inseticidas, com os nomes técnicos e comerciais, grupos químicos e dosagens utilizadas.

\begin{tabular}{llll}
\hline & & Dosagem & \\
& Nome técnico & Grupo químico & \\
\hline Thiodan 350 CE & endosulfan & 1,750 & éster do ac. sulf. diol cíclico \\
Lorsban 480 CE & chlorpyrifos & 1,200 & organofosforado \\
Turbo 50 CE & betacyfluthrin & 0,013 & piretróide \\
Kumulus 800 PM & enxofre & 4,000 & enxofre \\
Peropal 250 PM & azocyclotin & 0,310 & organo estânico \\
Cuprogarb 500 PM & oxicloreto de cobre & 5,000 & cúprico \\
\hline
\end{tabular}

fórmula $\mathrm{E}=100 \%-(100 \%-\mathrm{M} \%) \times \mathrm{R} 1 \times \mathrm{R} 2$, proposta por VOGT (1992), sendo: $\mathrm{E}=$ efeito total (\%); M\% = mortalidade no tratamento corrigida pela fórmula de ABBOTT (1925); R1 = razão entre a média diária de ovos colocados por fêmea tratada e não tratada e R2 = razão entre a viabilidade média de ovos postos por fêmea tratada e não tratada. Após a obtenção do efeito total, cada composto foi enquadrado em uma das quatro classes de toxicidade propostas pela "International Organization fot Biological and Integrated Control of Noxious Animals and Plants" - IOBC (HASSAN, 1997; STERK et al. 1999), sendo: classe 1 = inócuo (E<30\%), classe $2=$ levemente nocivo $(30 \leq \mathrm{E} \leq 79 \%)$, classe $3=$ moderadamente nocivo $(80 \leq \mathrm{E} \leq 99 \%)$ e classe $4=$ nocivo (E>99\%).

Os dados foram submetidos à análise de variância e as médias comparadas pelo teste de agrupamento de Scott - Knott a 5\% de significância (SCOTT \& KNOTT, 1974).

Aplicação dos produtos em adultos.

Dez casais de $\boldsymbol{C}$. externa com até 24 horas de idade, oriundos de uma criação de manutenção na quarta geração do laboratório, foram anestesiados com $\mathrm{CO}_{2}$ durante um minuto. Após serem anestesiados, os adultos foram colocados em placas de Petri de $15 \mathrm{~cm}$ de diâmetro por $2 \mathrm{~cm}$ de altura e pulverizados por meio de torre de Potter. Em seguida, foram individualizados, por casal, em gaiola de PVC de $10 \mathrm{~cm}$ de diâmetro e $10 \mathrm{~cm}$ de altura e mantidos nas mesmas condições climáticas e de alimentação empregadas no ensaio anterior.

O delineamento experimental foi inteiramente casualizado, com sete tratamentos e dez repetições, sendo cada parcela constituída por um casal de $\boldsymbol{C}$. externa. Após 24 horas da pulverização foi avaliada a mortalidade e a capacidade diária e total de oviposição durante quatro semanas consecutivas. A viabilidade dos ovos e o efeito total (E) foram determinados conforme ensaio com pupas.
Os dados referentes ao período de préoviposição, oviposição diária e total e a viabilidade de ovos foram transformados para $\sqrt{x+1}$ e submetidos à análise de variância, e as médias comparadas pelo teste Scott - Knott a 5\% de probabilidade (SCOTT \& KNOTT, 1974).

\section{RESULTADOS E DISCUSSÃO}

Efeito dos produtos fitossanitários sobre pupas de $\boldsymbol{C}$. externa.

A duração da fase de pupa de $\boldsymbol{C}$. externa que receberam aplicação dos produtos não foi afetada pelos compostos avaliados, variando de 10,5 a 10,9 dias (Tabela 2). A sobrevivência das pupas foi afetada por chlorpyrifos, com média de $77,5 \%$, diferindo dos demais tratamentos em que a sobrevivência foi de $90 \%$ a $97,5 \%$ (Tabela 2). A maior mortalidade causada por esse produto foi devida, provavelmente, ao seu modo de ação, por ingestão e/ou contato (RIGITANO \& CARVALHO, 2001), bem como pelo maior efeito residual, provocando a morte de adultos faratos (recém emergidos) quando entraram em contato com o composto, por ocasião do rompimento e saída do casulo.

Pupas pulverizadas com betacyfluthrin tiveram uma sobrevivência de 97,5\% (Tabela 2) assemelhando-se aos resultados obtidos por ULHÔA et al. (2002) e GODOY et al. (2004) quando pulverizaram pupas de C. externa com deltamethrin, esfenvalerato e fenpropathrin.

A razão sexual de adultos provenientes das pupas não foi afetada por nenhum dos compostos avaliados, variando de 0,47 a 0,55 (Tabela 2), estando de acordo aos resultados de GODOY et al. (2004) que observaram razão sexual de 0,5 para adultos de $\boldsymbol{C}$. externa, criadas a $25^{\circ} \mathrm{C}$.

Analisando-se o efeito total dos produtos avaliados sobre as pupas e os adultos de C. externa

Ciência Rural, v.36, n.1, jan-fev, 2006. 
Tabela 2 - Duração (dias) e sobrevivência (\%) ( \pm EP) de pupas e razão sexual ( \pm EP) de adultos de Chrysoperla externa (Hagen, 1861) (Neuroptera: Chrysopidae), provenientes de pupas pulverizadas com produtos fitossanitários $\left(25 \pm 2^{\circ} \mathrm{C}\right.$, UR de $70 \pm 10 \%$ e $12 \mathrm{~h}-$ luz/dia)

\begin{tabular}{llll}
\hline \multirow{2}{*}{ Tratamentos } & \multicolumn{2}{c}{ Fase de pupa } & \multirow{2}{*}{ Razão sexual } \\
\cline { 2 - 3 } & Duração & Sobrevivência & \\
\hline Endosulfan & $10,6 \pm 0,09 \mathrm{a}$ & $95,0 \pm 3,33 \mathrm{a}$ & $0,47 \pm 0,05 \mathrm{a}$ \\
Chlorpyrifos & $10,5 \pm 0,08 \mathrm{a}$ & $77,5 \pm 4,49 \mathrm{~b}$ & $0,55 \pm 0,06 \mathrm{a}$ \\
Betacyfluthrin & $10,6 \pm 0,11 \mathrm{a}$ & $97,5 \pm 2,50 \mathrm{a}$ & $0,47 \pm 0,07 \mathrm{a}$ \\
Enxofre & $10,9 \pm 0,13 \mathrm{a}$ & $95,0 \pm 3,33 \mathrm{a}$ & $0,47 \pm 0,06 \mathrm{a}$ \\
Azocyclotin & $10,6 \pm 0,13 \mathrm{a}$ & $90,0 \pm 5,53 \mathrm{a}$ & $0,52 \pm 0,05 \mathrm{a}$ \\
Oxicloreto de cobre & $10,7 \pm 0,10 \mathrm{a}$ & $97,5 \pm 5,52 \mathrm{a}$ & $0,52 \pm 0,06 \mathrm{a}$ \\
Testemunha & $10,9 \pm 0,18 \mathrm{a}$ & $97,5 \pm 2,50 \mathrm{a}$ & $0,53 \pm 0,05 \mathrm{a}$ \\
& & & \\
Média Geral & 10,7 & 92,9 & 0,5 \\
CV $(\%)$ & 2,83 & 11,99 & 37,71 \\
\hline
\end{tabular}

Médias não seguidas pela mesma letra nas colunas diferem significativamente entre si pelo teste de Scott - Knott em nível de 5\% de probabilidade de erro.

provenientes de pupas tratadas, os produtos endosulfan, betacyfluthrin, enxofre, azocyclotin e oxicloreto de cobre foram enquadrados na classe 1 (inócuos, $\mathrm{E}<30 \%$ ) e chlorpyrifos na classe 2 (levemente nocivo, $30 \leq \mathrm{E} \leq 79 \%$ ) (Tabela 3 ). Observou-se que quando $\boldsymbol{C}$. externa encontrava-se no estágio de pupa, sofreu pouca influência dos inseticidas testados. Isso ocorreu, provavelmente, pelo impedimento físico e mecânico à penetração dos compostos através da seda do casulo.

Esses resultados aproximam-se aos de GODOY et al. (2004) que, avaliando os efeitos de abamectin, lufenuron, fenbutatin oxide, tebufenozide e deltamethrin aplicados em pupas de C. externa, verificaram que a sobrevivência variou de 96,7 a $100 \%$ para essa espécie de predador. Também no caso de ULHÔA et al. (2002), ao avaliarem a seletividade de endosulfan, esfenvalerate, fenpropathrin, trichlorfon e triflumuron, encontraram sobrevivência de 71,7 a 100\%. Esses resultados fazem analogia, ainda, às observações de KOWALSKA \& SZCZEPANSKA (1988) que, ao testarem os efeitos de vários produtos para Chrysoperla carnea (Stephens, 1836), em diferentes fases do desenvolvimento, constataram que a fase de pupa foi a mais tolerante, possivelmente pela proteção proporcionada pela seda que forma o casulo.

Efeito dos produtos fitossanitários sobre adultos de C.externa.

Observou-se que chlorpyrifos e betacyfluthrin foram altamente nocivos aos adultos, ocasionando $100 \%$ e $82,5 \%$ de mortalidade, respectivamente, diferindo dos demais produtos. Observaram-se, ainda, 2,5\% de mortalidade para adultos pulverizados com azocyclotin e oxicloreto de cobre e de $0 \%$ para aqueles tratados com endosulfan, enxofre e na testemnha (Tabela 3 ).

Os resultados observados com o piretróide betacyfluthrin que proporcionou $82.5 \%$ de mortalidade dos adultos de C.externa (Tabela 3), são semelhantes àqueles de GODOY et al. (2004), que constataram para o piretróide deltamethrin, mortalidade de $100 \%$ de adultos de $\boldsymbol{C}$. externa. Igualmente àqueles de MATTIOLI et al. (1992) e SANTA-CECÍLIA et al. (1997) que, ao pulverizarem adultos de $\boldsymbol{C}$. cubana com deltamethrin, verificaram $100 \%$ de mortalidade.

Os adultos de $\boldsymbol{C}$. externa pulverizados com o piretróide betacyfluthrin sofreram um choque inicial "knock down", permanecendo estáticos por aproximadamente 24 horas, e, após esse período, 17,5\% dos indivíduos se recuperaram e reiniciaram a alimentação. Essas observações assemelham-se às de MORAES \& CARVALHO (1993) que constataram, após o choque inicial quando ficaram paralisados, a recuperação de $17,9 \%$ dos adultos do crisopídeo Ceraeochrysa cubana (Hagen, 1861) quando foram pulverizados com o piretróide fenpropathrin na dose de $0,4 \mathrm{ml} . \mathrm{L}^{-1}$ de água. Essas observações coincidem com a sintomatologia descrita por FERREIRA et al. (1993), quando aplicaram os piretróides fenpropathrin e bifenthrin sobre larvas de $\boldsymbol{C}$. cubana que além do efeito de choque, observaram paralisação de $40 \%$ das larvas por um período que variou de 4 a 18 horas. Segundo RIGITANO \& CARVALHO (2001), os piretróides possuem efeito de choque acentuado, porém pode ocorrer, posteriormente, a recuperação do inseto.

A sobrevivência de $97,5 \%$ dos adultos de

C. externa tratados com azocyclotin, observada nessa pesquisa (Tabela 3), compara-se à sobrevivência de $100 \%$ dos adultos de $\boldsymbol{C}$. cubana quando pulverizados com o acaricida fenbutatin oxide, do mesmo grupo químico dos organoestânicos, conforme observado por MATTIOLI et al. (1992) e SANTA-CECÍLIAet al. (1997).

Quanto à classe de toxicidade, endosulfan, enxofre, azocyclotin e oxicloreto de cobre foram enquadrados na classe 1 = inócuos $(\mathrm{E}<30 \%)$, betacyfluthrin na classe $3=$ moderadamente nocivo $(80 \leq \mathrm{E} \leq 99 \%)$ e chlorpyrifos na classe $4=$ nocivo (E>99\%) (Tabela 3). O efeito total provocado pelo acaricida azocyclotin é comparável àquele observado por GODOY et al. (2004) para fenbutatin oxide, do mesmo grupo químico.

Ciência Rural, v.36, n.1, jan-fev, 2006. 
Tabela 3 - Porcentagem de mortalidade, número médio de ovos/fêmea/dia, viabilidade dos ovos (\%), efeito total (E) e classe de toxicidade para alguns compostos aplicados a pupas e adultos de Chrysoperla externa (Hagen, 1861) (Neuroptera: Chrysopidae) (25 $\pm 2^{\circ} \mathrm{C}$, UR de $70 \pm 10 \%$ e $12 \mathrm{~h}-$ luz/dia).

\begin{tabular}{|c|c|c|c|c|c|c|c|}
\hline Tratamentos & Número de pupas & $\mathrm{M} \%{ }^{1}$ & $\mathrm{Mc}^{2}$ & $\mathrm{R}^{3}$ & $\mathrm{R} " \%{ }^{4}$ & $\mathrm{E} \%^{5}$ & Classe $^{6}$ \\
\hline Endosulfan & 40 & 5,0 & 1,3 & 17,36 & 93,65 & 7,14 & 1 \\
\hline Chlorpyrifos & 40 & 22,5 & 18,4 & 16,37 & 89,55 & 30,93 & 2 \\
\hline Betacyfluthrin & 40 & 2,5 & 0,0 & 15,65 & 93,91 & 19,04 & 1 \\
\hline Enxofre & 40 & 5,0 & 1,3 & 16,57 & 94,58 & 9,79 & 1 \\
\hline Azocyclotin & 40 & 10,0 & 5,3 & 17,25 & 95,37 & 9,98 & 1 \\
\hline Oxicloreto de cobre & 40 & 2,5 & 0,0 & 18,97 & 97,11 & $-4,94$ & 1 \\
\hline Testemunha & 40 & 2,5 & - & 17,80 & 97,65 & - & - \\
\hline Tratamentos & Número de adultos & $\mathrm{M} \%^{1}$ & $\mathrm{Mc}^{2}$ & $\mathrm{R}^{3}$ & $\mathrm{R} " \%{ }^{4}$ & $\mathrm{E} \%{ }^{5}$ & Classe $^{6}$ \\
\hline Endosulfan & 20 & 0,0 & 0,0 & 12,9 & 90,4 & 25,9 & 1 \\
\hline Chlorpyrifos & 20 & 100,0 & 100,0 & - & - & 100,0 & 4 \\
\hline Betacyfluthrin & 20 & 82,5 & 82,5 & - & - & 82,5 & 3 \\
\hline Enxofre & 20 & 0,0 & 0,0 & 15,1 & 92,7 & 9,8 & 1 \\
\hline Azocyclotin & 20 & 2,5 & 2,5 & 12,9 & 92,7 & 25,5 & 1 \\
\hline Oxicloreto de cobre & 20 & 2,5 & 2,5 & 15,6 & 92,4 & 11,1 & 1 \\
\hline Testemunha & 20 & 0,0 & - & 16,5 & 95,0 & - & - \\
\hline
\end{tabular}

${ }^{1}$ Mortalidade (\%) acumulada obtida ao longo do desenvolvimento do predador.

${ }^{2}$ Mortalidade (\%) corrigida pela fórmula de ABBOTT (1925).

${ }^{3}$ Número médio de ovos/dia/fêmea.

${ }^{4}$ Viabilidade (\%) dos ovos coletados no período de quatro semanas consecutivas.

${ }^{5}$ Efeito total (\%) dos tratamentos ao longo do desenvolvimento do predador.

${ }^{6}$ Classe de toxicidade preconizada pela IOBC (HASSAN, 1997; STERK et al., 1999), sendo: classe $1=$ inócuo $($ E<30\%), classe $2=$ levemente nocivo $(30 \leq \mathrm{E} \leq 79 \%)$, classe $3=$ moderadamente nocivo $(80 \leq \mathrm{E} \leq 99 \%)$ e classe $4=$ nocivo $(\mathrm{E}>99 \%)$.

Avaliando-se a capacidade reprodutiva de casais de C. externa sobreviventes, constatou-se que os produtos aplicados não afetaram o período de préoviposição, que variou de 5 a 5,2 dias (Tabela 4). Estes resultados são semelhantes aos obtidos por FIGUEIRA et al. (2002) que, estudando a biologia de adultos de $\boldsymbol{C}$. externa, observaram duração de 5,1 dias para o período de pré-oviposição de fêmeas alimentadas com a mesma dieta utilizada no presente estudo.

Considerando a oviposição média diária no período avaliado, observou-se que as fêmeas de $\boldsymbol{C}$. externa pulverizadas com endosulfan e azocyclotin apresentaram menor oviposição diária, com média de 12,9 ovos/fêmea/dia, em relação àquelas pulverizadas com enxofre, oxicloreto de cobre e água (testemunha), que apresentaram médias de 15,1; 15,6 e 16,5 ovos/ fêmea/dia, respectivamente (Tabela 4). Esses resultados foram próximos aos de FIGUEIRA et al. (2002), que obtiveram, em média, 18,5 ovos/fêmea/dia para adultos de $\boldsymbol{C}$. externa que não receberam nenhuma pulverização e foram alimentados com a mesma dieta.

Endosulfan e azocyclotin não afetaram a sobrevivência dos adultos, contudo influenciaram de alguma forma na reprodução, reduzindo a fecundidade. Esses resultados aproximam-se aos de ULHÔA et al. (2002), que constataram redução na capacidade de oviposição de $\boldsymbol{C}$. externa após receberem aplicação de triflumuron na dose de 0,038 g i.a. $\mathrm{L}^{-1}$ de água. VELLOSO et al. (1999) ao avaliarem inseticidas reguladores de crescimento sobre adultos desse mesmo crisopídeo, observaram que buprofezin, pyriproxifen e cyromazine não afetaram a capacidade de oviposição nem a viabilidade dos ovos, demonstrando que esses compostos podem atuar de maneira diferente na fisiologia de adultos dessa espécie, em relação ao composto triflumuron avaliado por ULHÔA et al. (2002).

A viabilidade dos ovos não foi afetada por nenhum produto, com médias variando de 90,1 a 94,6\% (Tabela 4), assemelhando-se aos resultados de FIGUEIRA et al. (2002) que obtiveram $87,7 \%$ de viabilidade para ovos de $\boldsymbol{C}$. externa que não recebeu nenhuma pulverização e foi alimentada com a mesma dieta e também aos de RIBEIRO et al. (1991), que observaram viabilidade de $95,4 \%$ para ovos dessa mesma espécie, que não recebeu nenhuma pulverização e foi alimentada com a mesma dieta. 
Tabela 4 - Período de pré-oviposição (em dias), oviposição diária, total e viabilidade (\%) ( \pm EP) de ovos provenientes de adultos de Chrysoperla externa (Hagen, 1861) (Neuroptera: Chrysopidae), pulverizados com alguns produtos fitossanitários $\left(25 \pm 2{ }^{\circ} \mathrm{C}\right.$, UR de $70 \pm 10 \%$ e $12 \mathrm{~h}-$ luz/dia).

\begin{tabular}{|c|c|c|c|c|}
\hline \multirow{2}{*}{ Tratamentos } & \multirow{2}{*}{ Período de Pré-oviposição ${ }^{1}$} & \multicolumn{2}{|c|}{ Oviposição ${ }^{2,}$} & \multirow{2}{*}{ Viabilidade } \\
\hline & & Diária & Total & \\
\hline Endosulfan & $5,1 \pm 0,18$ & $12,9 \pm 0,23 b$ & $360,0 \pm 1,27 b$ & $90,1 \pm 1,81$ \\
\hline Enxofre & $5,1 \pm 0,23$ & $15,1 \pm 0,13 \mathrm{a}$ & $422,1 \pm 0,72 \mathrm{a}$ & $92,7 \pm 0,74$ \\
\hline Azocychlotin & $5,2 \pm 0,20$ & $12,9 \pm 0,14 b$ & $360,1 \pm 0,76 \mathrm{~b}$ & $92,7 \pm 0,74$ \\
\hline Oxicloreto de cobre & $5,0 \pm 0,21$ & $15,6 \pm 0,10 \mathrm{a}$ & $435,9 \pm 0,52 \mathrm{a}$ & $92,4 \pm 0,64$ \\
\hline Testemunha & $5,0 \pm 0,21$ & $16,5 \pm 0,07 \mathrm{a}$ & $463,0 \pm 0,38 \mathrm{a}$ & $94,6 \pm 0,68$ \\
\hline Média Geral & 5,1 & 14,6 & 408,2 & 92,5 \\
\hline Teste F & $0,162^{\mathrm{ns}}$ & $2,519^{* *}$ & $2,544^{* *}$ & $2,465^{\mathrm{ns}}$ \\
\hline $\mathrm{CV}(\%)$ & 12,93 & 11,68 & 12,49 & 3,51 \\
\hline
\end{tabular}

${ }^{1}$ As médias nas colunas não diferem entre si pelo teste $\mathrm{F}$ a $5 \%$ de significância.

${ }^{2}$ As médias não seguidas das mesmas letras na coluna diferem entre si pelo teste de Scott - Knott em nível de 5\% de probabilidade de erro.

\section{CONCLUSÕES}

O composto chlorpyrifos é tóxico para pupas e adultos de $\boldsymbol{C}$. externa. Betacyfluthrin, endosulfan, enxofre, azocyclotin e oxicloreto de cobre são seletivos para pupas de $\boldsymbol{C}$. externa e não afetam a fase subseqüente de desenvolvimento desse crisopídeo, sendo betacyfluthrin tóxico para adultos de $\boldsymbol{C}$. externa, enquanto endosulfan, azocyclotin, enxofre e oxicloreto de cobre são seletivos. Em função da baixa toxicidade apresentada por endosulfan, azocyclotin, enxofre e oxicloreto de cobre, esses compostos podem ser recomendados em programas de manejo de pragas do cafeeiro em associação $\operatorname{com} \boldsymbol{C}$. externa.

\section{AGRADECIMENTOS}

À Fundação de Amparo à Pesquisa do Estado de Minas Gerais (FAPEMIG) pela concessão de bolsa de doutorado, e à Universidade Federal de Lavras (UFLA) e Epamig CTSM/ EcoCentro pelos suportes laboratoriais.

\section{REFERÊNCIAS}

ABBOTT, W.S. A method of computing the effectiveness of an insecticide. Journal of Economic Entomology, Lanham, v.18, p.265-267, 1925.

BARBOSA, L.R. et al. Capacidade reprodutiva e viabilidade de ovos de Ceraeochrysa everes (Banks, 1920) (Neuroptera: Chrysopidae) em diferentes condições de acasalamento. Ciência e Agrotecnologia, Lavras, v.26, n.3, p.466-471, 2002 .

CARVALHO, C.F.; SOUZA, B. Métodos de criação e produção de crisopídeos. In: BUENO, V.H.P. (Ed). Controle biológico de pragas: produção massal e controle de qualidade. Lavras: UFLA, 2000. Cap.6, p.91-109.

FERREIRA, M.N. et al. Seletividade de acaricidas para larvas de Ceraeochrysa cubana (Hagen, 1861) (Neuroptera: Chrysopidae), em laboratório. Ciência e Prática, Lavras, v.17, n.1, p.71-77, 1993.

FIGUEIRA, L.K. et al. Influência da temperatura sobre alguns aspectos biológicos de Chrysoperla externa (Hagen, 1861) (Neuroptera: Chrysopidae) alimentada com ovos de Alabama argillacea (Hübner, 1818) (Lepidoptera: Noctuidade). Ciência e Agrotecnologia, Lavras, v.26, p.1439-1450, 2002. (Edição Especial).

FONSECA, A.R. et al. Capacidade predatória e aspectos biológicos das fase imaturas de Chrysoperla externa (Hagen, 1861) (Neuroptera: Chrysopidae) alimentada com Schizaphis graminum (Rondani, 1852) (Hemiptera: Aphididae) em diferentes temperaturas. Ciência e Agrotecnologia, Lavras, v. 25, n.2, p.251-263, 2001 .

GLIESSMAN, S.R. Agroecologia - processos ecológicos em agricultura sustentável. (Tradução-Maria José Guazzelli). Porto Alegre, RS: UFRGS, 2000. 653p.

GODOY, M.S. et al. Seletividade de Seis Inseticidas Utilizados em Citros a Pupas e adultos de Chrysoperla externa (Hagen, 1861) (Neuroptera: Chrysopidae). Ciência e Agrotecnologia, Lavras, v.33, p.359-364, 2004.

GRAFTON-CARDWELL, E.E.; HOY, M.A. Short-term effects of permethrin and fenvalerate on oviposition by Chrysoperla carnea (Neuroptera: Chrysopidae). Journal of Economic Entomology, Lanham, v.78, n.4, p.955-959, 1985 .

HASSAN, S.A. Métodos padronizados para testes de seletividade, com ênfase em Trichogramma. In: PARRA, J.R.P.; ZUCCHI, R. (Ed). Trichogramma e o controle biológico aplicado. Piracicaba: FEALQ, 1997. p.207-233. 
KOWALSKA, T.; SZCZEPANSKA, K. Effect of pesticides on Chrysopidae. In: NIEMCZYK, E.; DIXON, A.F.G. Ecology and effectiveness of aphidophaga. Hague: SPB Academic, 1988. p.333-336.

MATTIOLI, E. et al. Efeitos de inseticidas e acaricidas sobre ovos, larvas e adultos do predador Ceraeochrysa cubana (Hagen, 1861) (Neuroptera: Chrysopidae) em laboratório. Ciência e Prática, Lavras, v.16, n.4, p.491-497, 1992.

MORAES, J.C.; CARVALHO, C.F. Seletividade de acaricidas a ovos, larvas e adultos de Ceraeochrysa cubana (Hagen, 1861) (Neuroptera: Chrysopidae). Ciência e Prática, Lavras, v.17, n.4, p.388-392, 1993.

REIS, P.R. et al. Manejo ecológico das principais pragas do cafeeiro. Informe Agropecuário, Belo Horizonte, v.23, n.214/ 215, p.83-99, 2002.

RIBEIRO, M.J. et al. Influência da alimentação larval sobre a biologia de adultos de Chrysoperla externa (Hagen, 1861) (Neuroptera: Chrysopidae). Ciência e Prática, Lavras, v.15, n.2, p.349-354, 1991.

RIGitano, R.L.O.; CARVAlHO, G.A. Toxicologia e seletividade de inseticidas. Lavras: UFLA/FAEPE, 2001. $72 \mathrm{p}$.

SANTA-CECÍLIA, L.V.C. et al. Seletividade de alguns inseticidas/acaricidas aos adultos de Ceraeochrysa cubana
(Hagen, 1861) (Neuroptera: Chrysopidae) em laboratório. Pesquisa Agropecuária Brasileira, Brasília, v.32, n.8, p.803806, 1997.

SCOTT, A.J.; KNOTT, M.A. A cluster analyses method for grouping means in the analyses of variance. Biometrics, Washington, v.30, n.3, p.502-512, 1974.

STERK, G. et al. Results of the seventh joint pesticide testing programme carried out by the IOBC/WPRS-Working Group 'Pesticides and Beneficial Organisms'. Bio Control, v.44, p.99-117, 1999.

VELLOSO, A.H.P.P. et al. Efeitos de compostos reguladores de crescimento de insetos sobre larvas e adultos de Chrysoperla externa (Hagen, 1861) (Neuroptera: Chrysopidae). Ciência e Agrotecnologia, Lavras, v.23, n.1, p.96-101, 1999.

VOGT, H. Untersuchungen zu nebenwirkungen von insektiziden und akariziden auf Chrysoperla carnea (Stephens) (Neuroptera: Chrysopidae). Mededelingen Rijksfaacuteit Landbouwwetenschappen te Gent, Gent, v.57, p.559-567, 1992.

ULHÔA, J.L.R. et al. Ação de inseticidas recomendados para o controle do curuquerê-do-algodoeiro para pupas e adultos de Chrysoperla externa (Hagen, 1861) (Neuroptera: Chrysopidae). Ciência e Agrotecnologia, Lavras, v.26, p.1365-1372, 2002. (Edição Especial). 